\title{
THE SIGNIFICANCE OF ABNORMALITIES IN THE FORM OF THE ELECTROCARDIOGRAM *
}

\author{
G. CANBY ROBINSON, M.D. \\ ST. LOUIS
}

Much interest has recently been shown in the relation of changes in the form of the electrocardiogram to disturbances of the myocardium. This interest is centered for the most part about changes in the form of the ventricular portion of the electrocardiogram, which have been interpreted as indicative of faulty conduction of the cardiac impulse through the ventricles. The abnormal form of the electrocardiogram, to which attention is directed in this paper, consists in a prolongation of the initial group of waves of the ventricular portion, the so-called Q-R-S group, with notching or splitting of the main wave, the $\mathrm{R}$ wave. A consideration of this portion of the electrocardiogram is important, as it renders valuable information concerning the state of the myocardium, and has proved of distinct value in determining the diagnosis and prognosis in numerous cases of heart disease.

It is a well established fact that the electrical phenomenon accompanying cardiac activity, which is expressed by the electrocardiogram, begins before the actual contraction of the heart muscle, and the first series of waves constituting the ventricular portion of the electrocardiogram represents, in part, the spread of the cardiac impulse into and through the ventricles. A disturbance in the passage of cardiac impulse along the intraventricular conducting system and through the ventricular musculature causes a change in the form of the first portion of the ventricular curve. The prolongation of the Q-R-S group indicates that the time required for the complete activation of the ventricles is lengthened, and the notching probably indicates that the activation of the various portions of the ventricles is not taking place in its proper sequence. This interpretation of the type of abnormal electrocardigram which has been mentioned is generally accepted. The purpose of this paper is to present some facts which apparently throw light on the mechanism by which the conduction into and through the ventricles is sometimes disturbed, and an interpretation of the abnormal electrocardiogram is offered which differs from that which has been generally expressed.

* From the Department of Internal Medicine, Washington University School of Medicine. 
In a paper which I published in $1916,{ }^{1}$ electrocardiograms were reproduced which demonstrated that the type of abnormal electrocardiogram which is here under discussion may result when the stimulus of the heart beat reaches the ventricles before they have fully recovered from previous activity and when they are in a state which may be conveniently termed functional fatigue. It was pointed out in this paper that under these circumstances the disturbance of the intravenous conduction is dependent on the fact that an insufficient time has elapsed for the recovery of the ventricles and the curves reproduced show that when a sufficient length of time has elapsed the following electrocardiogram has a form which is much nearer that yielded by the normal heart. This is especially well seen in a record from a case of sino-auricular block in which an idioventricular contraction usually occurs during the latter part of the pause caused by the block. This idioventricular contraction precedes by a short interval the auricular beat, which sends down a stimulus to the ventricles, stimulating them soon after the completion of the idioventricular contraction. The interval of time elapsing between the idioventricular contraction and the stimulation of the ventricles by the impulse sent down by the auricular contraction varies, and the form of the ventricular complex produced by the second ventricular activity also varies in form. A study of a large series of records from this case shows that the form of the complex yielded by the second ventricular contraction bears a direct relationship to the period of rest that precedes it. When the period of rest is very short the ventricles fail to respond to the impulse descending from the auricles. When the period of rest is somewhat longer, the ventricles respond, but yield a complex with a much prolonged Q-R-S group and with well defined notching of the $R$ wave. When the period of rest is still further prolonged the ventricular complex approaches the normal complex in form. This is shown in Figure 1 of the previous paper.

The records of this case demonstrate, therefore, that so-called functional fatigue may be responsible for the abnormal form of the ventricular complex under discussion, and in this instance an anatomic lesion can hardly be considered as playing a rôle in its production.

In the paper that has been mentioned records of a case of auricular fibrillation were reproduced in which the same abnormalities of form are seen in the ventricular portion of the electrocardiogram whenever the diastolic pause preceding the ventricular contraction is relatively short, while following longer pauses the ventricular complex assumes

1. Robinson, G. C.: The Relation of Changes in the Form of the Ventricular Complex of the Electrocardiogram to Functional Changes in the Heart, Arch. Int. Med. 18:830 (Dec.) 1916. 
a form much nearer the normal. The interpretation seems justified that the disturbance in conduction through the ventricles in this case is dependent on lack of time for recovery, and that when sufficient time elapses between beats, intraventricular conduction apparently takes place at a relatively normal rate and in a relatively normal manner. (Figs. 4 and 5 of the previous paper.)

The evidence of these two cases and others reported with them has led to the belief that in cases in which the ventricular complexes constantly show certain abnormal forms there may be functional changes in the heart which prevent the normal recovery of intraventricular conduction during diastole. This explanation for the cause of the abnormalities of the electrocardiogram under discussion has not, however, been emphasized by those who have since published papers on this subject. Thus Oppenheimer and Rothschild ${ }^{2}$ have attempted to correlate gross and histologic changes in the hearts of several patients with the abnormal form of electrocardiogram without considering the possibility that physiologic rather than anatomic changes might be responsible for the altered form of the electrocardiograms. Although they mention the cases showing temporary changes in the electrocardiograms, they seem to consider that they belong to another type of case from those which they describe. This division of the cases does not seem to me to be justified.

Following this paper by Oppenheimer and Rothschild there seems to have been a general acceptance in this country of the idea, either stated or implied, that abnormal forms of electrocardiograms are indicative of intraventricular block caused by localized subendocardial lesions. This is to be noted in the papers of Blackford, Willius and Haines, ${ }^{3}$ Neuhof, ${ }^{4}$ Carter ${ }^{5}$ and Willius. ${ }^{6}$ Wedd, ${ }^{7}$ whose paper deals with less pronounced changes in the form of the electrocardiogram, recognizes, however, the possibility of functional derangement of intraventricular conduction without localized lesions in the conducting system. Willius goes so far as to state that "arborization block or impaired intraventricular conduction is dependent on graphic records

2. Oppenheimer, B. S., and Rothchild, M. A.: Electrocardiographic Changes Associated with Myocardial Involvement, J. A. M. A. 69:429 (Aug. 11) 1917.

3. Blackford, J. M., Willius, F. A., and Haines, L. B.: Operative Risk in Cardiac Disease, J. A. M. A. 69:2011 (Dec. 15) 1917.

4. Neuhof, S.: The Clinical Significance of the Abnormally Wide Ventricular Deviation in the Electrocardiogram, Arch. Int. Med. 22:45 (July) 1918.

5. Carter, E. P.: Further Observations on the Aberrant Electrocardiogram Associated with Sclerosis of the Atrio-Ventricular Bundle Branches and Their Terminal Arborization, Arch. Int. Med. 22:331 (Sept.) 1918.

6. Willius, F. A.: Arborization Block, Arch. Int. Med. 23:431 (April) 1919.

7. Wedd, A. M.: The Clinical Significance of Slight Notching of the $R$ Wave of the Electrocardiogram, Arch. Int. Med. 23:515 (April) 1919. 
for its recognition. It is now generally accepted to indicate disease of the subendocardial myocardium and evidence of serious functionai cardiac disturbance. The involvement occurs in the subendocardial or Perkinje plexus." This statement is made in spite of the fact that the postmortem examination of five hearts revealed no definite localizing lesions, but rather diffuse degenerative processes involving the myocardium.

Carter has reported a case yielding abnormal electrocardiograms in which a very careful histologic study of the subendocardial structures was made. He considers that there existed a causal relation between the abnormal electrocardiograms and the sclerosis that we found in the atrioventricular bundle branches and their terminal arborizations. In this case the explanation of the abnormal electrocardiograms seems well grounded, although not definitely proved. There was found a discrepancy between the amount and position of the sclerotic changes in the ventricular conducting system and the anticipated form of the electrocardiogram, suggesting that some other factor than the anatomic lesion may have been operative.

Fridericia and M $\phi 1 l e{ }^{8}$ also report a case showing abnormal electrocardiograms in which there was an extensive lesion in the intraventricular system causing, the authors consider, intraventricular block.

I have recently had an opportunity of studying a case which seems to throw some new light on the causation of the abnormal form of electrocardiograms, which I wish to report before considering the underlying causes of the disturbance of intraventricular conduction.

\section{CASE REPORT}

A man, 63 years of age, a telegraph operator, entered the hospital with severe symptoms of cardiac decompensation, showing marked dyspnea, edema of the extremities and cyanosis. His history revealed no definite etiology, but there was an unsubstantiated history of syphilis. $\mathrm{He}$ had had cardiac symptoms, especially palpitation, for about a year, and had dyspnea for six months, with frequent attacks of nocturnal dyspnea. From the examination a diagnosis of arterial sclerosis, chronic myocarditis, auricular fibrillation and mitral insufficiency were made. Electrocardiograms obtained May 10, 1917, showed the ventricular rate to be 135 per minute, and the form of the ventricular portion was abnormal in all leads, the initial group of waves being prolonged to 0.170 to 0.180 second (maximum normal $=0.100 \mathrm{sec}$.) and definite notching was present. The form of the electrocardiogram suggested right branch bundle block, but was not typical, and indicated a high degree of derangement in the intraventricular conducting system (Fig. 1).

This patient was given a single dose of a standardized tincture of digitalis, 6 c.c., the dose being smaller than that usually administered in cases of auricular fibrillation in this clinic, because of a history of having taken the drug in small doses up to the time of admission to the hospital. The patient responded

8. Fridericia and M $\phi$ ller, P.: Et Tilfaelde af Myocarditis, lokaliseret til septum Ventriculorum, med ejendommelize abnormiter; Elaktrokaardiogrammet, Hospitalstid. 60:573 (June 13) 1917; 60:597 (June 20) 1917. 
remarkably well to the drug and electrocardiograms obtained two days later showed a ventricular rate of 69 per minute. The form of the ventricular complexes was strikingly changed. The initial group of waves had a duration of from 0.92 to 0.10 second, and the notching of the main wave had almost entirely disappeared (Fig. 2). The clinical improvement was also striking. No more digitalis was administered at this time and the ventricular rate gradually increased. Electrocardiograms obtained May 13 and May 15, showed a rate of 85 per minute, with increased arrhythmia (Fig. 3). In the records the ventricular complexes have distinctly more notching and prolongation of the initial group of waves than that obtained when the ventricular rate was 69 per minute. A feature of these records is the definite abnormality of form seen in the complexes yielded by the ventricular contractions which are preceded by short diastolic periods. Under these conditions the complexes have a form approaching that seen when the heart was beating at a rate of 135 per minute.

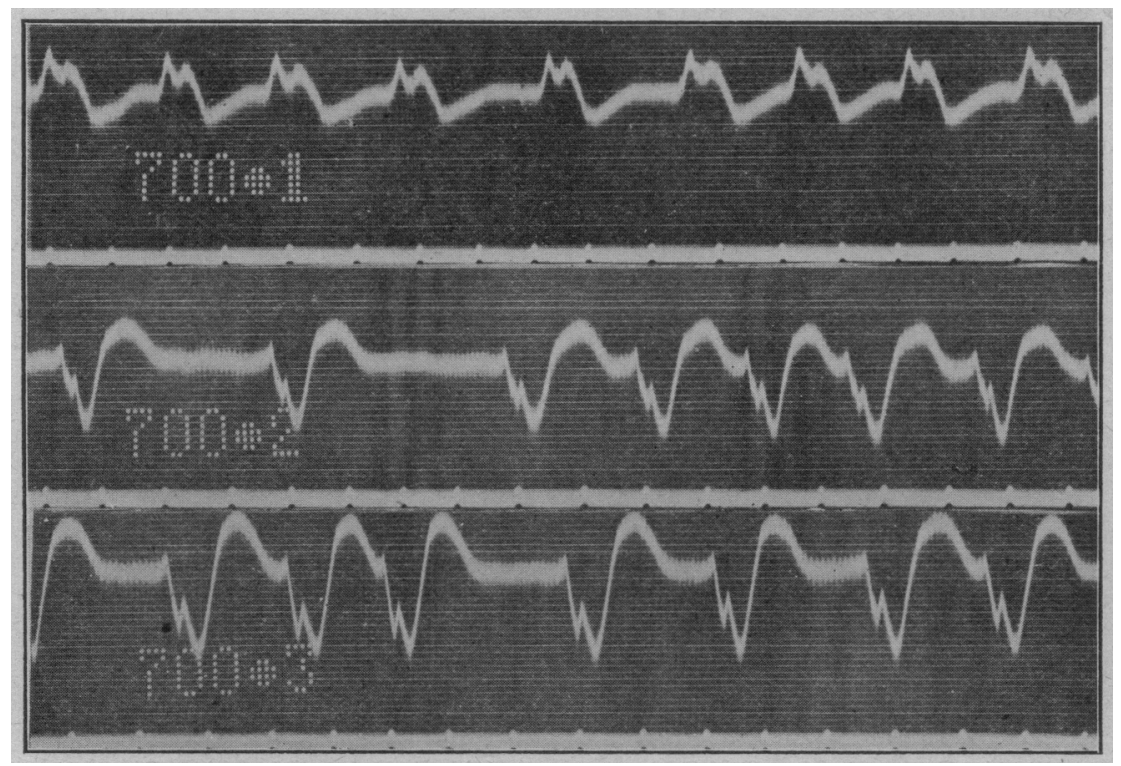

Fig. 1.-Electrocardiogram, three usual leads, obtained May 10, 1917, when patient first entered the hospital. Ventricular rate, 135 per minute. Initial group of waves of ventricular complex from 0.170 to 0.180 second in duration.

The administration of digitalis again slowed the heart and caused a disappearance of the electrocardiographic evidence of disturbance of intraventricular conduction. Cardiac efficiency became much improved and the patient left the hospital with instructions to use the tincture of digitalis constantly. These instructions were not carried out, however, and the patient returned six weeks later with slight signs of cardiac inefficiency and with a ventricular rate of from 144 to 158 beats per minute. At this time, however, the electrocardiograms failed to reveal any prolongation of the initial waves of the ventricular complexes, and only slight notching was present (Fig. 4). In other words, at this time, in spite of a higher ventricular rate than that first seen, there was practically no evidence of a disturbance of intraventricular conduction. The use of the tincture of digitalis again slowed the heart, and a record obtained one month after the second admission showed a ventricular rate of 61 beats per minute, with practically no change in the form of the electrocardiograms. 
It is difficult to conceive how the disturbance in the intraventricular conduction which was evidently present in this case when first observed could have been caused by an anatomical lesion involving the subendocardial tissues and the arborization of the ventricular conductory system, because of its rapid disappearance when the heart rate became slowed by digitalis. The fact that the disturbance of intraventricular conduction, the so-called arborization block, did not return later with the increase in ventricular rate also indicates that some improvement in the physiologic processes of the heart had occurred during the period of treatment.

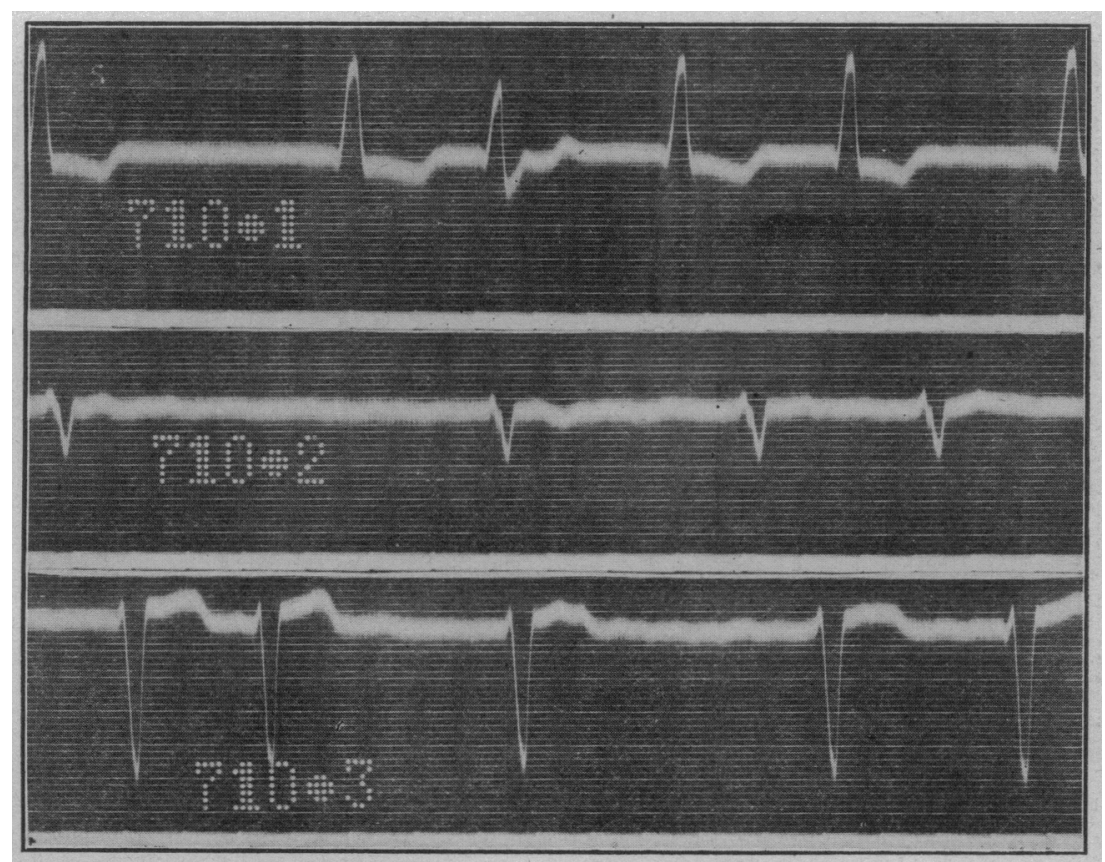

Fig. 2.-Electrocardiogram obtained May 12, 1917, after a single dose of digitalis had been given. Ventricular rate 69 per minute. Striking change in form of complexes. Initial group of waves of ventricular complex from 0.092 to 0.100 second in duration.

A consideration of this case as well as of those that have alreaciy been reported and reviewed brings out the necessity for another explanation than that which had been offered by Oppenheimer and Rothschild and others to account for the abnormalities in the form of the electrocardiograms seen in these cases.

The term functional fatigue has already been used to account for the derangement of intraventricular conduction. Of course, a recognized change in structure may bear a causative relation to a derange- 
ment in a physiologic process, but in the light of the cases that I have reported this association can by no means be taken for granted, and other so-called physiologic factors must come into play in these cases.

I wish to discuss briefly the possible physiologic factors that seem to me likely to play a rôle in the production of disturbances in intraventricular conduction, and which in turn may be worthy of consideration as a factor in the production of cardiac inefficiency in cases of heart disease.

In the cases under consideration an improvement in the state of the intraventricular conduction takes place during the diastolic periods when the heart is at rest. But instances are seen when the diastolic period is not long enough to allow complete recovery, and in the case reported in this paper the recovery of intraventricular conduction is slower at

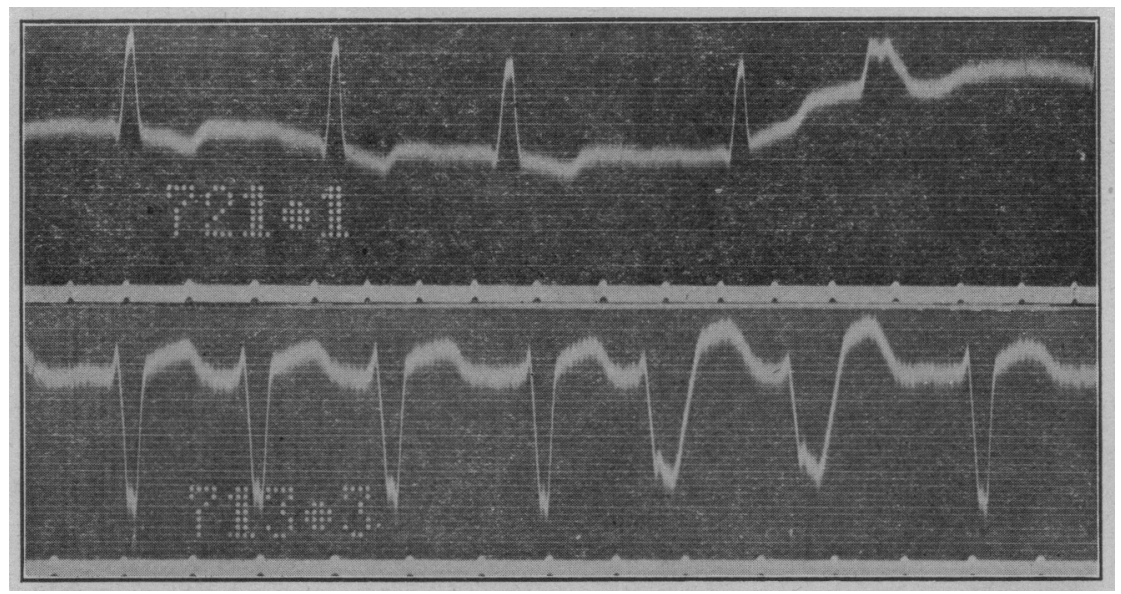

Fig. 3.-Electrocardiograms obtained May 13 and May 15, 1917. Ventricular rate, 85 per minute. The form of the ventricular complexes and the duration of the initial group of waves depend on the length of the preceding diastole. First and third leads are shown.

one time than at another. It may be inferred from these observations that certain metabolic processes occur during ventricular activity which affect the function of the conducting mechanism, and that a definite time is required for the recovery of the ventricles before the conducting of the cardiac impulse proceeds through them in a normal manner. The length of time required for this recovery may be increased distinctly in damaged hearts, and under certain conditions the diastolic rest may be continually insufficient to allow complete recovery of the intraventricular conducting system. This is apparently the cause of the abnormal electrocardiograms shown in Figure 1. Figure 3 shows, however, that the degree of recovery of the conducting system is 
dependent on the length of the cardiac rest afforded by the diastolic period.

I wish to suggest that the derangement of intraventricular conduction which is indicated by the abnormal form of the electrocardiogram may be caused in these cases by the presence of acid metabolites, and that these acid metabolites are present in increased amounts when there is a deficient supply of oxygen to the tissues involved.

The basis for this explanation is the work of Fletcher and Hopkins $^{9}$ on the effect of diminished oxygen supply on muscle activity and the work of Mines $^{10}$ and of Burridge ${ }^{11}$ on the effects of changes in hydrogen ion concentration on conduction through the ventricles.

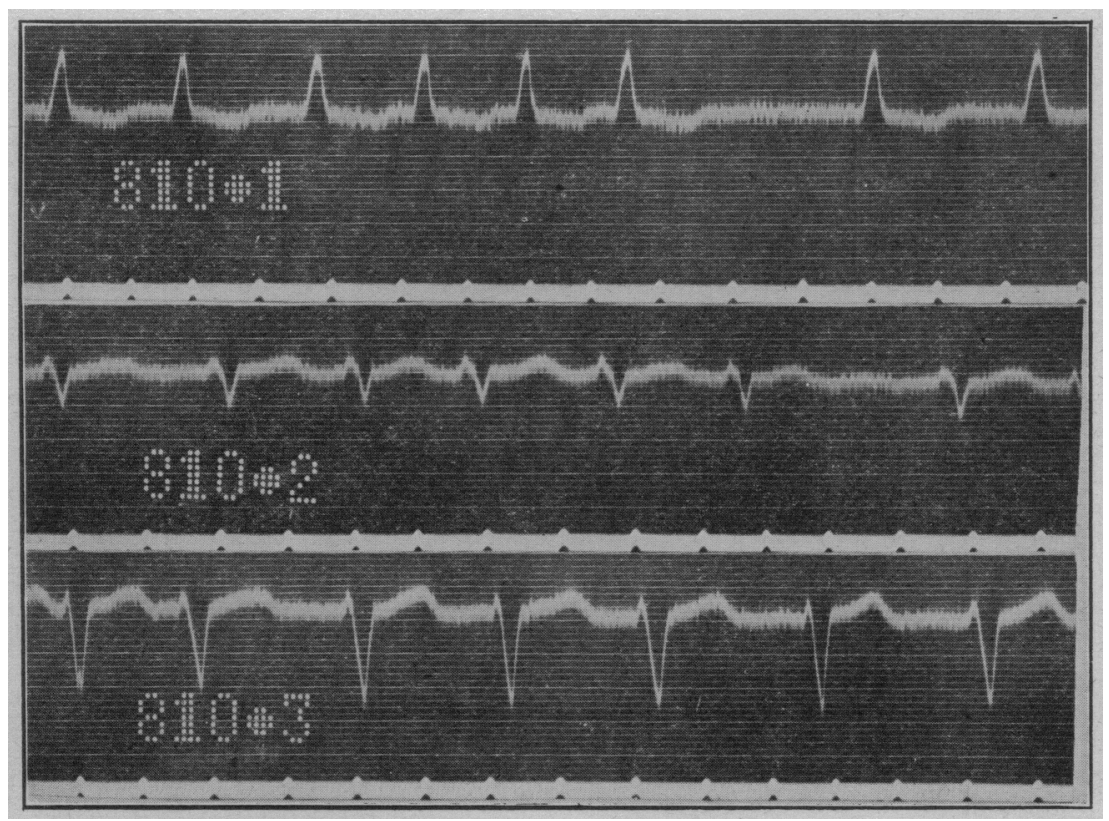

Fig. 4.--Electrocardiogram obtained June 29, 1917. The ventricular rate is 158 per minute, but the abnormalities of the ventricular cemplexes previously seen do not occur.

Fletcher and Hopkins have carried out an investigation on lactic acid in amphibian muscle, and state that there is a large body of wellknown evidence which indicates that the disposal of acid products whatever the site of it may be - is most efficient when conditions for

9. Fletcher and Hopkins: Lactic Acid in Amphibian Muscle, J. Physiol. $\mathbf{3 5}: 247,1907$.

10. Mines: On Dynamic Equilibrium in the Heart, J. Physiol. 46:349, 1913.

11. Burridge: Propagation of Contraction in the Frog's Heart, J. Physiol. $45: 6,1912$. 
oxidative processes are most favorable, and that it is incomplete when these conditions are unfavorable. Their experiments show that the lactic acid formed in muscle follows this rule, and its disappearance from muscle is dependent on the oxygen supply.

With these facts in mind, it is not difficult to conceive that acid metabolites tend to accumulate in the heart muscle when the oxygen supply is insufficient to meet the demands of the rapidly beating heart. In a number of the hearts showing delayed intraventricular conduction marked sclerosis of the coronary arteries have been observed, notably in the series of Oppenheimer and Rothschild ${ }^{2}$ in which twelve of the fourteen hearts examined postmortem showed well defined coronary artery sclerosis. This lesion may, therefore, be considered responsible for an inadequate oxygen supply to the heart muscle, which results in an excessive production of acid metabolites.

Burridge $^{x I}$ has shown that the addition of acid to the fluid with which the frog's heart was perfused caused a delay in the conduction of the impulse. He states further that if sufficient acid were added to cause the heart to stop beating, sodium bicarbonate neutralization brought back the beat, but with markedly delayed conduction of the impulse through the ventricle. Mines ${ }^{10}$ has used these experiments to account for certain changes in the electrocardiogram which result from increased frequency of the heart beat which he believes may lead to changes in the hydrogen ion concentration in some region of the muscle. If these experiments can be considered as holding good for the chemical changes that occur during the cardiac action of the human heart, they seem to justify the hypothesis that any change which diminishes the oxygen supply to the heart muscle will favor the accumulation of acid metabolites and consequently cause a derangement in the propagation of the cardiac impulse through the ventricles. This derangement would become apparent whenever the diastolic rest is of insufficient duration to allow the removal of the acid metabolites from the ventricular structures. Sclerosis of the coronary arteries would furnish the condition for the increased production of acid metabolites and also tend to delay their removal. Myocardial lesions, such as a fibrosis, would perhaps also tend to produce these effects by lessening the efficiency of the blood supply to the ventricular structures.

It should be stated that an objection to the foregoing hypothesis may be raised in that there is no evidence that acid metabolites are produced by the activity of the conducting system. Erlanger ${ }^{12}$ was unable to discover the production of acid in the conducting system as a result of its activity. The effect of acid produced in the muscle on conduction is therefore open to question.

12. Erlanger, J.: Personal communication. 


\section{SUMMARY}

Certain abnormalities in the form of the electrocardiogram are recognized as evidence of faulty conduction of the cardiac impulse through the ventricles. These abnormalities consist of a prolongation of the time required to complete the first series of waves, the Q-R-S group of the ventricular part of the electrocardiogram and notching of the main wave of the group. An attempt has been made by several writers to associate faulty conduction of the cardiac impulse within the ventricles with anatomical lesions involving the intraventricular conducting system. This association may be correctly assumed in some cases, but in this paper cases are reported which seem to prove that faulty conduction through the ventricles may depend on what has been termed "functional fatigue." Examples are cited in which derangement of ventricular conduction has resulted from lack of time for conduction recovery after a ventricular contraction. In the case reported distinct improvement in ventricular conduction followed slowing of the heart rate, when longer diastolic pauses were present. The rate of recovery of conduction was observed also in this case to become more rapid with improved circulatory efficiency.

The hypothesis is advanced that in the cases referred to in this paper the disturbance of intraventricular conduction is the result of the presence of acid metabolites in the ventricular structures, and that sclerosis of the coronary arteries and perhaps other lesions causing an insufficient blood supply to the ventricles are responsible for the presence of the acid metabolites, as it has been shown that these accumulate whenever the oxygen supply to muscle is inadequate. This hypothesis renders an anatomic lesion in the subendocardial structures unnecessary to account for the disturbance of the intraventricular conduction, although the effect of acid produced in the muscle on conduction is still open to question. 